\title{
Micromechanics and effective elastic moduli of particle-reinforced composites with near-field particle interactions
}

Received: 16 February 2010 / Published online: 25 May 2010

(C) The Author(s) 2010. This article is published with open access at Springerlink.com

\begin{abstract}
A micromechanical framework is proposed to predict effective elastic moduli of particle-reinforced composites. First, the interacting eigenstrain is derived by making use of the exterior-point Eshelby tensor and the equivalence principle associated with the pairwise particle interactions. Then, the near-field particle interactions are accounted for in the effective elastic moduli of spherical-particle-reinforced composites. On the foundation of the proposed interacting solution, the consistent versus simplified micromechanical field equations are systematically presented and discussed. Specifically, the focus is upon the effective elastic moduli of two-phase composites containing randomly distributed isotropic spherical particles. To demonstrate the predictive capability of the proposed micromechanical framework, comparisons between the theoretical predictions and the available experimental data on effective elastic moduli are rendered. In contrast to higher-order formulations in the literature, the proposed micromechanical formulation can accommodate the anisotropy of reinforcing particles and can be readily extended to multi-phase composites.
\end{abstract}

\section{Introduction}

Due to their higher structural efficiency and versatility, composite materials have been widely studied and employed in diverse fields of science and engineering disciplines. In comparison with many conventional materials (such as steel and aluminum), fiber- or particle-reinforced composites offer salient features, such as low density, high strength-to-weight ratio, high stiffness, high toughness, improved creep resistance, enhanced wear resistance, superior environmental durability, custom microstructure-morphology, and preferred directionality. The matrix material may consist of metal, ceramic, or polymer, for example. The inclusions encompass unidirectionally aligned, bi-directional, or randomly dispersed particles/fibers in a matrix material. While the continuous-fiber reinforced composites provide the most effective strengthening in a given direction, the particle-reinforced composites could be attractive due to their cost-effectiveness, feasible isotropic properties, and the ability to be processed with similar manufacturing processes to monolithic materials.

For predicting the effective properties of composites, analytical models based on Eshelby's approach [1] are popular. This aims at the analysis of a representative volume element (RVE) or area element (RAE). The major advantage of Eshelby's approach is that it enables us to predict full multi-axial properties and responses

\author{
J. W. Ju $(\varangle) \cdot$ K. Yanase \\ Department of Civil and Environmental Engineering, University of California, \\ Los Angeles, CA 90095-1593, USA \\ E-mail: juj@ucla.edu \\ URL: http://www.cee.ucla.edu/faculty/ju.htm \\ Present Address: \\ K. Yanase \\ Department of Mechanical Engineering, Fukuoka University, Fukuoka, Japan
}


of heterogeneous materials. Based on the pioneering work by Eshelby, the so-called effective medium theories (the Eshelby method, the Mori-Tanaka method, the self-consistent method, the differential scheme, the generalized self-consistent method, and so on) are widely employed to predict effective properties of heterogeneous materials. Nevertheless, these effective medium theories are based on the single-inclusion problem; only the average effects of all other inclusions (i.e., far-field interactions) are considered (cf. [2]). Accordingly, in order to predict the deformation responses of composites with high volume fractions, the individual or near-field inclusion interactions need to be adequately accounted for.

To tackle the near-field inclusion interaction problem, several approaches were proposed in the literature. In the presence of near-field interactions, the eigenstrain may no longer be constant. Therefore, Moschovidis and Mura [3] formulated the polynomial eigenstrain to account for the effects of interactions for the stress/strain fields. In practice, it is almost impossible to find the eigenstrain in the presence of many inclusion interactions. To overcome this issue, Ju and Chen [4-6] developed an approximate, yet accurate method to characterize the inter-particle interaction effects in a two-phase composite. Specifically, the Ju-Chen's higher-order (in terms of particle-volume fraction) micromechanical formulation can be applied to modeling composites with higher volume fractions. Further, Ju and Zhang [7,8] formulated the higher-order micromechanical formulation for continuous-fiber reinforced composites on the foundation of Ju and Chen's higher-order formulation. More recently, Lin and Ju [9] extended the Ju-Chen's pairwise interaction formulation to three-phase composites containing many randomly dispersed, isotropic spherical particles.

The objective of the present paper is to extend Ju-Chen's micromechanical framework [4-6] to predict effective elastic moduli of spherical-particle-reinforced composites in conjunction with the near-field particle interactions. The proposed approach is based on the probabilistic particle interaction solution within the micromechanical framework and homogenization. Namely, by making use of Eshelby's equivalence principle, effective elastic moduli of composites are analytically derived with the interacting eigenstrain caused by the near-field particle interactions.

\section{Effective elastic moduli of multi-phase composites}

\subsection{Pairwise particle interaction}

Let us consider the interaction caused by the pair of particles as illustrated in Fig. 1. In the presence of direct particle interactions, the following equation may no longer hold:

$$
\boldsymbol{\varepsilon}^{\prime}=\mathbf{S}: \varepsilon^{* *}
$$

where

$$
S_{i j k l}=\frac{1}{15\left(1-v_{0}\right)}\left[\left(5 v_{0}-1\right) \delta_{i j} \delta_{k l}+\left(4-5 v_{0}\right)\left(\delta_{i k} \delta_{j l}+\delta_{i l} \delta_{j k}\right)\right] .
$$

Here, $\boldsymbol{\varepsilon}^{* *}$ is the eigenstrain, and $\mathbf{S}$ is the interior-point Eshelby tensor for a spherical inclusion [10,11]. Moreover, $v_{0}$ represents the Poisson's ratio of the surrounding isotropic matrix material. In contrast to Eq. (1), the perturbed strain in $\Omega^{I}$ can be affected by the presence of an additional particle as follows (cf. Fig. 1):

$$
\boldsymbol{\varepsilon}^{I^{\prime}}(\mathbf{x})=\mathbf{S}^{I}: \varepsilon^{I^{* *}}+\overline{\mathbf{G}}^{I I}(\overline{\mathbf{x}}): \varepsilon^{I I^{* *}}
$$

where $\overline{\mathbf{G}}(\overline{\mathbf{x}})$ represents the exterior-point Eshelby's tensor (cf. [6,10,12-16]) for a spherical particle expressed with the $\overline{\mathbf{x}}$ coordinate system. The components of $\overline{\mathbf{G}}$ can be expressed as [6]:

$$
\begin{aligned}
\overline{\mathbf{G}}(r)= & \frac{\rho^{3}}{30\left(1-v_{0}\right)}\left[\left(3 \rho^{2}-10 v_{0}+5\right)\left(\delta_{i k} \delta_{j l}+\delta_{i l} \delta_{j k}\right)+\left(3 \rho^{2}+10 v_{0}-5\right) \delta_{i j} \delta_{k l}\right. \\
& +15\left(1-\rho^{2}\right) \delta_{i j} n_{k} n_{l}+15\left(1-2 v_{0}-\rho^{2}\right) \delta_{k l} n_{i} n_{j}+15\left(7 \rho^{2}-5\right) n_{i} n_{j} n_{k} n_{l} \\
& \left.+15\left(v_{0}-\rho^{2}\right)\left(\delta_{i k} n_{j} n_{l}+\delta_{i l} n_{j} n_{k}+\delta_{j k} n_{i} n_{l}+\delta_{j l} n_{i} n_{k}\right)\right]
\end{aligned}
$$

where $\rho=a / r, a$ is the particle radius, $r$ represents the center-to-center distance of particles, and $\delta$ signifies the Kronecker delta. Furthermore, by making use of the spherical coordinate system, the components of the unit outward normal vector $\mathbf{n}$ can be rendered as:

$$
n_{1}=\cos \phi \cdot \cos \theta, \quad n_{2}=\cos \phi \cdot \sin \theta, \quad n_{3}=\sin \phi .
$$




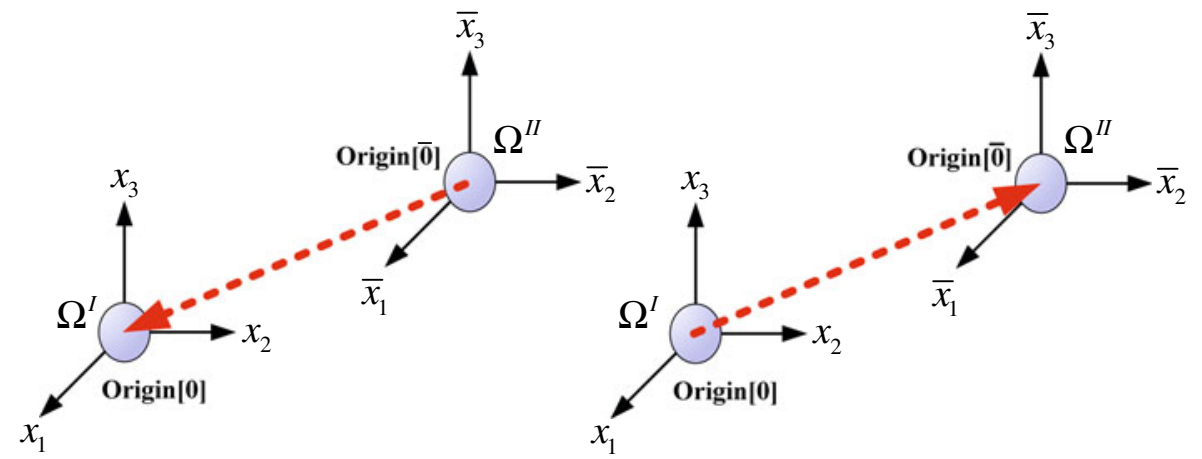

Fig. 1 The pairwise particle interactions

In some types of composite materials, there may exist residual stresses caused by the manufacturing process. For instance, due to the high temperature manufacturing process, thermal residual stresses can be generated in metal matrix composites. Such residual stresses can be effectively accommodated by introducing the thermal eigenstrain $\varepsilon^{*}$ [17-19]. Therefore, in the presence of thermal eigenstrain, the equivalence equation can be written as:

$$
\mathbf{C}_{0}:\left(\boldsymbol{\varepsilon}^{0}+\boldsymbol{\varepsilon}^{\prime}-\boldsymbol{\varepsilon}^{* *}\right)=\mathbf{C}^{I}:\left(\boldsymbol{\varepsilon}^{0}+\boldsymbol{\varepsilon}^{\prime}-\boldsymbol{\varepsilon}^{*}\right)
$$

Here, $\mathbf{C}_{0}$ and $\mathbf{C}^{I}$ are the fourth-order elastic stiffness of the matrix and the particle- $I$, respectively, and $\boldsymbol{\varepsilon}^{0}$ signifies the far-field applied strain. In contrast to the formulation proposed by Moschovidis and Mura [3], we consider the constant or volume-averaged total eigenstrain $\boldsymbol{\varepsilon}^{* *}$ for the sake of simplicity. Hence, by using Eqs. (3) and (6), the following equation can be obtained:

$$
\left(\Delta \mathbf{C}^{I} \bullet \mathbf{S}+\mathbf{C}_{0}\right): \boldsymbol{\varepsilon}^{I^{* *}}+\Delta \mathbf{C}^{I} \bullet \overline{\mathbf{G}}^{I I}(\overline{\mathbf{x}}): \boldsymbol{\varepsilon}^{I I^{* *}}=-\Delta \mathbf{C}^{I} \varepsilon^{0}+\mathbf{C}^{I}: \boldsymbol{\varepsilon}^{*}
$$

where

$$
\Delta \mathbf{C}^{I}=\mathbf{C}^{I}-\mathbf{C}_{0}
$$

In addition, by performing the volume-averaging in the $\Omega^{I}$ domain with a Taylor expansion [3,5] at the origin of the $\mathbf{x}$ coordinate (cf. Fig. 1), we obtain:

$$
\begin{aligned}
& \left(\Delta \mathbf{C}^{I} \bullet \mathbf{S}+\mathbf{C}_{0}\right): \boldsymbol{\varepsilon}^{I^{* *}}+\frac{1}{\Omega^{I}} \Delta \mathbf{C}^{I} \bullet\left\{\iint_{\Omega^{I}}\left(\overline{\mathbf{G}}^{I I}[0]+\frac{\partial \overline{\mathbf{G}}^{I I}[0]}{\partial \bar{x}_{m}} x_{m}+\frac{1}{2} \frac{\partial \overline{\mathbf{G}}^{I I}[0]}{\partial \bar{x}_{m} \partial \bar{x}_{n}} x_{m} x_{n}+\cdots\right) \mathrm{d} V\right\}: \boldsymbol{\varepsilon}^{I I^{* *}} \\
& =-\Delta \mathbf{C}^{I}: \boldsymbol{\varepsilon}^{0}+\mathbf{C}^{I}: \boldsymbol{\varepsilon}^{*} .
\end{aligned}
$$

Hence, we can write:

$$
\begin{aligned}
& \left(\Delta \mathbf{C}^{I} \bullet \mathbf{S}+\mathbf{C}_{0}\right): \boldsymbol{\varepsilon}^{I^{* *}}+\frac{1}{\Omega^{I}} \Delta \mathbf{C}^{I} \bullet\left\{\overline{\mathbf{G}}^{I I}[0] \Omega^{I}+\frac{\partial \overline{\mathbf{G}}^{I I}[0]}{\partial \bar{x}_{m}} \int_{\Omega^{I}} x_{m} \mathrm{~d} V+\frac{1}{2} \frac{\partial \overline{\mathbf{G}}^{I I}[0]}{\partial \bar{x}_{m} \partial \bar{x}_{n}} \int_{\Omega^{I}} x_{m} x_{n} \mathrm{~d} V+\cdots\right\}: \boldsymbol{\varepsilon}^{I I^{* *}} \\
& =-\Delta \mathbf{C}^{I}: \boldsymbol{\varepsilon}^{0}+\mathbf{C}^{I}: \boldsymbol{\varepsilon}^{*}
\end{aligned}
$$

where

$$
\int_{\Omega^{I}} x_{m} \mathrm{~d} V=0, \quad \int_{\Omega^{I}} x_{m} x_{n} \mathrm{~d} V=\frac{4 a_{I}^{5} \pi}{15} \delta_{m n}=\frac{a_{I}^{2}}{5} \Omega^{I} \delta_{m n}
$$

and $a_{I}$ represents the radius of the particle- $I$. 
By applying the Taylor expansion up to the second-order, one can obtain the following expression:

$$
\left(\Delta \mathbf{C}^{I} \bullet \mathbf{S}^{I}+\mathbf{C}_{0}\right): \boldsymbol{\varepsilon}^{I^{* *}}+\Delta \mathbf{C}^{I} \bullet \hat{\mathbf{G}}^{I I}: \boldsymbol{\varepsilon}^{I I^{*}}=-\Delta \mathbf{C}^{I}: \boldsymbol{\varepsilon}^{0}+\mathbf{C}^{I}: \boldsymbol{\varepsilon}^{*}
$$

In addition, by making use of the mean particle size (i.e., $\left.a^{I}=a^{I I}=a\right), \hat{\mathbf{G}}$ can be expressed as

$$
\begin{aligned}
\hat{\mathbf{G}}(r)= & \overline{\mathbf{G}}^{I I}[0]+\frac{a^{2} \cdot \delta_{m n}}{10} \frac{\partial \overline{\mathbf{G}}^{I I}[0]}{\partial \bar{x}_{m} \partial \bar{x}_{n}} \\
= & \frac{\rho^{3}}{30\left(1-v_{0}\right)}\left[\left(6 \rho^{2}-10 v_{0}+5\right)\left(\delta_{i k} \delta_{j l}+\delta_{i l} \delta_{j k}\right)+\left(6 \rho^{2}+10 v_{0}-5\right) \delta_{i j} \delta_{k l}\right. \\
& +15\left(1-\rho^{2}\right) \delta_{i j} n_{k} n_{l}+15\left(1-2 v_{0}-\rho^{2}\right) \delta_{k l} n_{i} n_{j}+15\left(14 \rho^{2}-5\right) n_{i} n_{j} n_{k} n_{l} \\
& \left.+15\left(v_{0}-2 \rho^{2}\right)\left(\delta_{i k} n_{j} n_{l}+\delta_{i l} n_{j} n_{k}+\delta_{j k} n_{i} n_{l}+\delta_{j l} n_{i} n_{k}\right)\right]+0\left(\rho^{8}\right) .
\end{aligned}
$$

It is noted that the $\rho^{7}$-order terms become zero, the order of the leading error in Eq. (13) is $\rho^{8}$.

Consequently, on the basis of Eq. (7), the equivalence equations for $\Omega^{I}$ and $\Omega^{I I}$ can be written as

$$
\begin{aligned}
\mathbf{A}^{I}: \boldsymbol{\varepsilon}^{I^{* *}}+\hat{\mathbf{G}}: \boldsymbol{\varepsilon}^{I I^{* *}} & =-\boldsymbol{\varepsilon}^{0}+\mathbf{B}^{I}: \boldsymbol{\varepsilon}^{I^{*}} \\
\hat{\mathbf{G}}: \boldsymbol{\varepsilon}^{I^{* *}}+\mathbf{A}^{I I}: \boldsymbol{\varepsilon}^{I I^{* *}} & =-\boldsymbol{\varepsilon}^{0}+\mathbf{B}^{I I}: \boldsymbol{\varepsilon}^{I I^{*}},
\end{aligned}
$$

where

$$
\mathbf{A}^{I}=\left(\Delta \mathbf{C}^{I}\right)^{-1} \bullet \mathbf{C}_{0}+\mathbf{S}, \quad \mathbf{B}^{I}=\left(\Delta \mathbf{C}^{I}\right)^{-1} \bullet \mathbf{C}^{I}
$$

After solving the above equations to find $\varepsilon^{I^{* *}}$, one can obtain the following relation:

$$
\left[\mathbf{A}^{I}-\hat{\mathbf{G}} \bullet\left(\mathbf{A}^{I I}\right)^{-1} \bullet \hat{\mathbf{G}}\right]: \boldsymbol{\varepsilon}^{I^{* *}}=\left[\hat{\mathbf{G}} \bullet\left(\mathbf{A}^{I I}\right)^{-1}-\mathbf{I}\right]: \boldsymbol{\varepsilon}^{0}+\mathbf{B}^{I}: \boldsymbol{\varepsilon}^{I^{*}}+\hat{\mathbf{G}} \bullet\left(\mathbf{A}^{I I}\right)^{-1} \bullet \mathbf{B}^{I I}: \boldsymbol{\varepsilon}^{I I^{*}}
$$

Here, I represents the fourth-order identity tensor.

2.2 Pairwise particle interaction with conditional probability

Let us consider the probable location for the second particle (cf. Fig. 2). Therefore, Eq. (17) leads to the following equation:

$$
\begin{aligned}
& {\left[\mathbf{A}^{I}-\int_{V \notin \Omega^{I}} \hat{\mathbf{G}} \bullet\left(\mathbf{A}^{I I}\right)^{-1} \bullet \hat{\mathbf{G}} \cdot P\left(\mathbf{x}^{I I} \mid \mathbf{x}^{I}\right) \mathrm{d} V\right]: \varepsilon^{I^{* *}}} \\
& =\left[\int_{V \notin \Omega^{I}} \hat{\mathbf{G}} \bullet\left(\mathbf{A}^{I I}\right)^{-1} \cdot P\left(\mathbf{x}^{I I} \mid \mathbf{x}^{I}\right) \mathrm{d} V-\mathbf{I}\right]: \varepsilon^{0}+\mathbf{B}^{I}: \varepsilon^{I^{*}} \\
& +\left[\int_{V \notin \Omega^{I}} \hat{\mathbf{G}} \bullet\left(\mathbf{A}^{I I}\right)^{-1} \bullet \mathbf{B}^{I I} \cdot P\left(\mathbf{x}^{I I} \mid \mathbf{x}^{I}\right) \mathrm{d} V\right]: \varepsilon^{I I^{*}} .
\end{aligned}
$$

Here, $P\left(\mathbf{x}^{I I} \mid \mathbf{x}^{I}\right)$ represents the conditional probability density function to find the second particle in the presence of the first particle. After integrating Eq. (18) in the spherical coordinate system, we arrive at:

$$
\left[\mathbf{A}^{I}-\int_{V \notin \Omega^{I}} \hat{\mathbf{G}} \bullet\left(\mathbf{A}^{I I}\right)^{-1} \bullet \hat{\mathbf{G}} \cdot P\left(\mathbf{x}^{I I} \mid \mathbf{x}^{I}\right) \mathrm{d} V\right]: \varepsilon^{I^{* *}}=-\boldsymbol{\varepsilon}^{0}+\mathbf{B}^{I}: \boldsymbol{\varepsilon}^{I^{* *}} .
$$




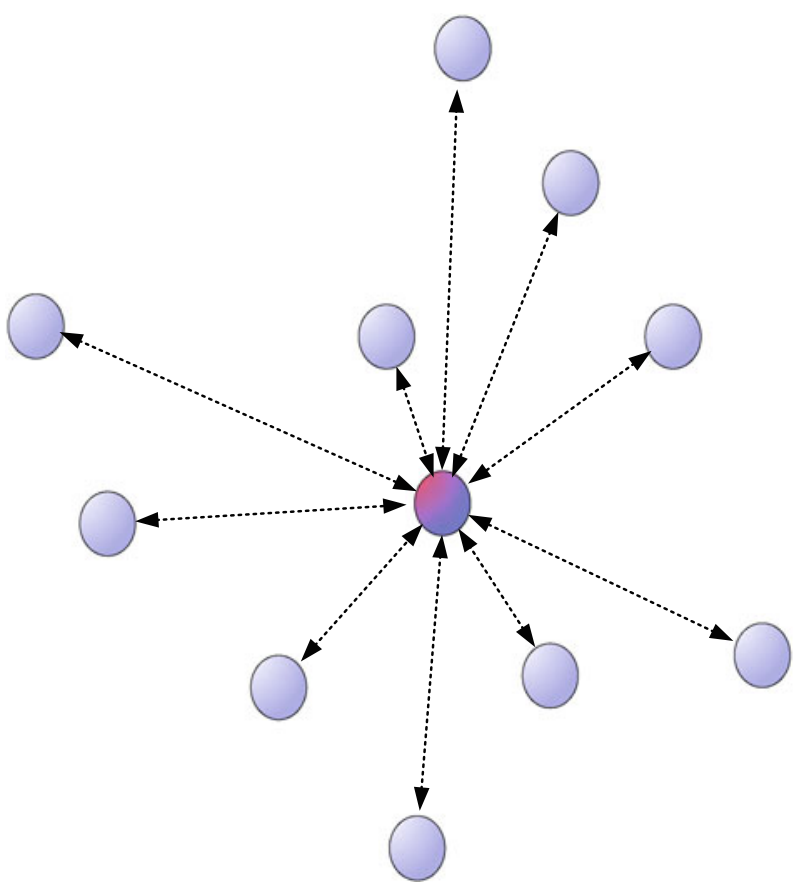

Fig. 2 The probabilistic pairwise particle interactions

It is noted that the fourth-order tensors $\overline{\mathbf{G}}$ and $\hat{\mathbf{G}}$ have the following properties:

$$
\begin{aligned}
& \int_{2 a}^{\infty}\left(\int_{0}^{2 \pi}\left(\int_{-\pi / 2}^{\pi / 2} \overline{\mathbf{G}} \cos \phi \mathrm{d} \phi\right) \mathrm{d} \theta\right) r^{2} \mathrm{~d} r=\mathbf{0}, \\
& \int_{2 a}^{\infty}\left(\int_{0}^{2 \pi}\left(\int_{-\pi / 2}^{\pi / 2} \hat{\mathbf{G}} \cos \phi \mathrm{d} \phi\right) \mathrm{d} \theta\right) r^{2} \mathrm{~d} r=\mathbf{0} .
\end{aligned}
$$

The above results can be obtained by taking advantage of the following relations:

$$
\begin{gathered}
\int_{0}^{2 \pi}\left(\int_{-\pi / 2}^{\pi / 2} n_{i} n_{j} \cos \phi \mathrm{d} \phi\right) \mathrm{d} \theta=\frac{4 \pi}{3} \delta_{i j}, \\
\int_{0}^{2 \pi}\left(\int_{-\pi / 2}^{\pi / 2} n_{i} n_{j} n_{k} n_{l} \cos \phi \mathrm{d} \phi\right) \mathrm{d} \theta=\frac{4 \pi}{15}\left(\delta_{i j} \delta_{k l}+\delta_{i k} \delta_{j l}+\delta_{i l} \delta_{j k}\right) .
\end{gathered}
$$

In particular, the result of Eq. (20) can be easily reproduced by the Tanaka-Mori lemma [20,21]. In practice, in the absence of actual manufacturing evidences, it is often assumed that the two-point conditional probability function is statistically isotropic, uniform and obeys the following form (cf. [5]):

$$
P\left(\mathbf{x}^{I I} \mid \mathbf{x}^{I}\right)=\left\{\begin{array}{cc}
N^{I I} / V, & \text { if } r>a \\
0, & \text { otherwise }
\end{array}\right\} .
$$

In Eq. (24), $V$ is the volume of $R V E, N / V$ signifies the number density of particles, and the following relation holds:

$$
\frac{N^{(r)}}{V}=\frac{3 \phi^{(r)}}{4 \pi a^{3}},
$$


where $\phi^{(r)}$ is the volume fraction of $r$ th - phase particles. It is noted that Eq. (25) can serve as the simplest approximation since it tends to underestimate the probabilistic existence of the second particle in the neighborhood of $\mathbf{x}^{I}$; particularly for the high particle-volume fractions. Therefore, Eq. (24) may be regarded as the lower bound for the microstructure. On the other hand, if we assume statistical isotropy, then the two-point conditional probability function depends on the radial distribution function $g(r)$ :

$$
P\left(\mathbf{x}^{I I} \mid \mathbf{x}^{I}\right)=\frac{N^{I I}}{V} g(r)
$$

Therefore, Eq. (19) can be rephrased as:

$$
\left\{\mathbf{A}^{I}-\frac{N^{I I}}{V} \int_{V \notin \Omega^{I}} \hat{\mathbf{G}} \bullet\left(\mathbf{A}^{I I}\right)^{-1} \bullet \hat{\mathbf{G}} \cdot g(r) \mathrm{d} V\right\}: \boldsymbol{\varepsilon}^{I^{* *}}=-\boldsymbol{\varepsilon}^{0}+\mathbf{B}^{I}: \boldsymbol{\varepsilon}^{I^{*}} .
$$

Finally, in the presence of several distinct properties of particles, we write the eigenstrain within each phase of particle as follows:

$$
\begin{gathered}
\left(\mathbf{A}^{(1)}-\sum_{r=1}^{N} \overline{\mathbf{A}}^{(r)}\right): \boldsymbol{\varepsilon}^{1^{* *}}=-\boldsymbol{\varepsilon}^{0}+\mathbf{B}^{(1)}: \boldsymbol{\varepsilon}^{1^{*}}, \\
\left(\mathbf{A}^{(2)}-\sum_{r=1}^{N} \overline{\mathbf{A}}^{(r)}\right): \boldsymbol{\varepsilon}^{2^{* *}}=-\boldsymbol{\varepsilon}^{0}+\mathbf{B}^{(2)}: \boldsymbol{\varepsilon}^{2^{*}}, \\
\left(\mathbf{A}^{(N)}-\sum_{r=1}^{N} \overline{\mathbf{A}}^{(r)}\right): \boldsymbol{\varepsilon}^{N^{* *}}=-\boldsymbol{\varepsilon}^{0}+\mathbf{B}^{(N)}: \boldsymbol{\varepsilon}^{N^{*}},
\end{gathered}
$$

where

$$
\overline{\mathbf{A}}^{(r)}=\frac{N^{(r)}}{V} \int_{V \notin \Omega^{(r)}} \hat{\mathbf{G}} \bullet\left(\mathbf{A}^{(r)}\right)^{-1} \bullet \hat{\mathbf{G}} \cdot g(r) \mathrm{d} V .
$$

In the case of $g(r)=1$, the components of the $\overline{\mathbf{A}}^{(r)}$ tensor can be obtained after lengthy but straightforward derivations (cf. Appendix I).

\subsection{Radial distribution function}

In computational mechanics and statistical mechanics, a radial distribution function (RDF, $g(r)$ ) describes how the density of the surrounding matter varies as a function of the distance from a particular point. Given a potential energy function, the radial distribution function can be found via computer simulations, such as the Monte Carlo method. It is also possible to use rigorous statistical mechanics to establish a suitable RDF. The Percus-Yevick approximation is a well-known solution for the radial distribution function (RDF) of a hard-sphere liquid. In the literature, a tractable form of solution is proposed by Trokhymchuk et al. [22] (cf. Appendix II).

In Fig. 3, the predicted RDFs are compared with the data predicted from the Monte Carlo simulation. As illustrated, reasonable fits are obtained for each volume fraction up to $\phi=0.471$. According to Fig. 3 , it is apparent that the assumption of the uniform radial distribution $g(r)=1$ is a reasonable approximation only for small volume fractions. 

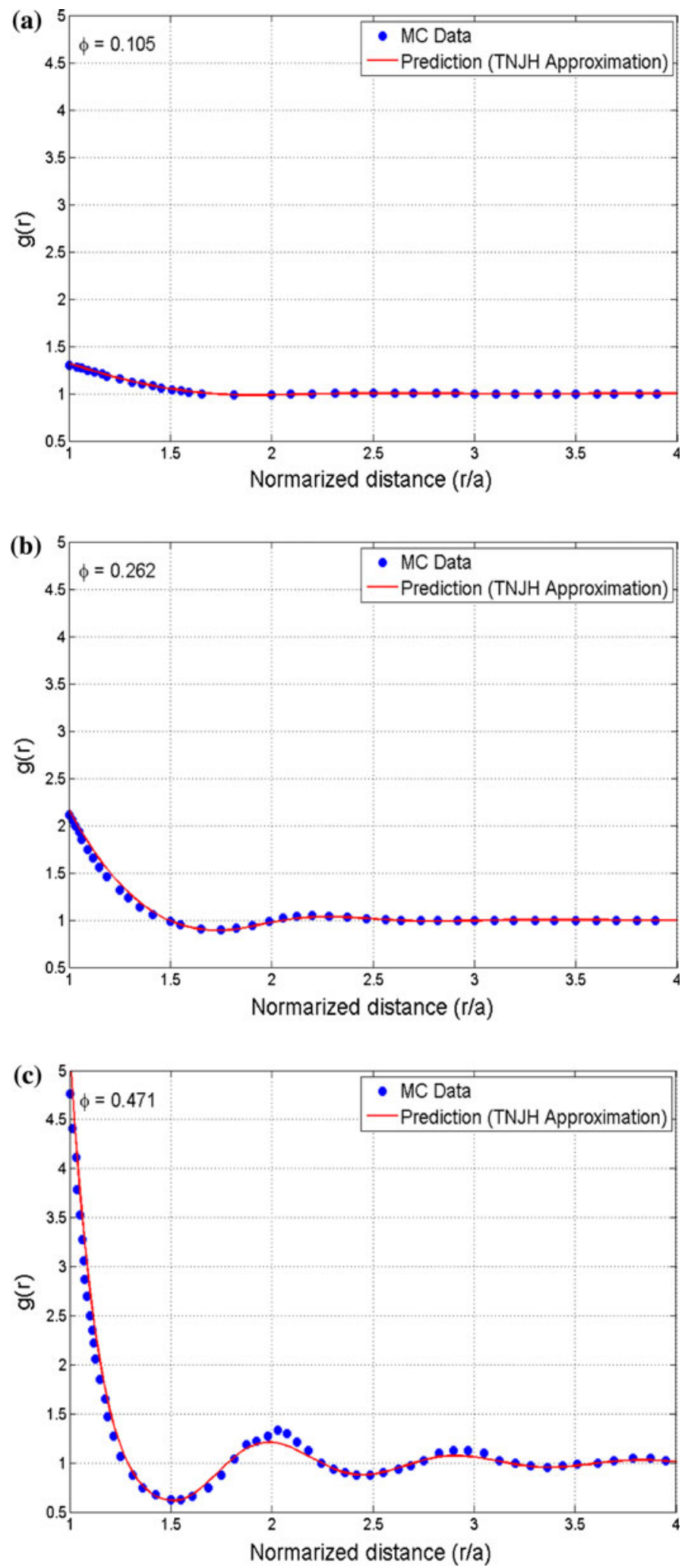

Fig. 3 The comparisons of radial distributions. a The volume fraction $\phi=0.105$. b The volume fraction $\phi=0.262$. $\mathbf{c}$ The volume fraction $\phi=0.471$ 
2.4 Consistent and simplified perturbed strains in spherical particles

Once the interacting eigenstrain is obtained by Eq. (28), one needs to find the perturbed strains within the particles. For instance, in the absence of residual stress, the perturbed strains in the particles within the two-phase composite can be obtained by using the equivalence equation consistently:

$$
\mathbf{C}_{0}:\left(\varepsilon^{0}+\boldsymbol{\varepsilon}^{\prime}-\boldsymbol{\varepsilon}^{* *}\right)=\mathbf{C}^{(1)}:\left(\varepsilon^{0}+\boldsymbol{\varepsilon}^{\prime}\right) .
$$

Therefore, by taking advantage of Eqs. (28) and (30), the consistent perturbed strain can be expressed as

$$
\boldsymbol{\varepsilon}^{\prime}=\mathbf{H}: \varepsilon^{0}
$$

where

$$
\mathbf{H}=\left(\Delta \mathbf{C}^{(1)}\right)^{-1} \bullet \mathbf{C}_{0} \bullet\left(\mathbf{A}^{(1)}-\overline{\mathbf{A}}^{(1)}\right)^{-1}-\mathbf{I}
$$

Since it is not a single-inclusion problem anymore, the interior-point Eshelby's tensor is not directly employed to find the perturbed strain in Eq. (31). Instead of using Eq. (31), the perturbed strain can also be approximated with the interior-point Eshelby tensor $\mathbf{S}$ (cf. Eq. (1)) for simplicity. Therefore, without resorting to the equivalence equation, one can obtain the following simplified perturbed strain:

$$
\boldsymbol{\varepsilon}^{\prime}=\mathbf{K}: \varepsilon^{0}
$$

where

$$
\mathbf{K}=-\mathbf{S} \bullet\left(\mathbf{A}^{(1)}-\overline{\mathbf{A}}^{(1)}\right)^{-1}
$$

2.5 Effective elastic stiffness of two-phase composites

We now consider the volume-averaged strain:

$$
\begin{aligned}
\overline{\boldsymbol{\varepsilon}} & =\boldsymbol{\varepsilon}^{0}+\frac{V_{m}}{V} \overline{\boldsymbol{\varepsilon}}_{m}^{\prime}+\frac{1}{V} \sum_{r=1}^{N} V_{r} \overline{\boldsymbol{\varepsilon}}_{r}^{\prime} \\
& \cong \boldsymbol{\varepsilon}^{0}+\frac{V_{m}}{V}\left[\frac{1}{V_{m}} \sum_{r=1}^{N}\left(\int_{\mathbf{x} \notin \Omega_{r}} \int_{\mathbf{y} \in \Omega_{r}} \mathbf{G}(\mathbf{x}-\mathbf{y}): \overline{\boldsymbol{\varepsilon}}_{r}^{* *}(\mathbf{y}) \mathrm{d} V(\mathbf{y}) \mathrm{d} V(\mathbf{x})\right)\right]+\frac{1}{V} \sum_{r=1}^{N} V_{r} \overline{\boldsymbol{\varepsilon}}_{r}^{\prime} \\
& =\boldsymbol{\varepsilon}^{0}+\frac{1}{V} \sum_{r=1}^{N} V_{r} \overline{\boldsymbol{\varepsilon}}_{r}^{\prime} \\
& =\boldsymbol{\varepsilon}^{0}+\sum_{r=1}^{N} \phi^{(r)} \overline{\boldsymbol{\varepsilon}}_{r}^{\prime} .
\end{aligned}
$$

Here, G represents the Green's function (cf. [2,10]), and the volume-averaged perturbed strains are considered. In addition, the Tanaka-Mori lemma [20] is applied for a spherical RVE with a spherical particle to solve the integration. The volume-averaged stress can be cast as (cf. [4]):

$$
\overline{\boldsymbol{\sigma}}=\mathbf{C}_{0}:\left[\overline{\boldsymbol{\varepsilon}}-\sum_{r=1}^{N} \phi^{(r)} \boldsymbol{\varepsilon}^{r^{* *}}\right] .
$$




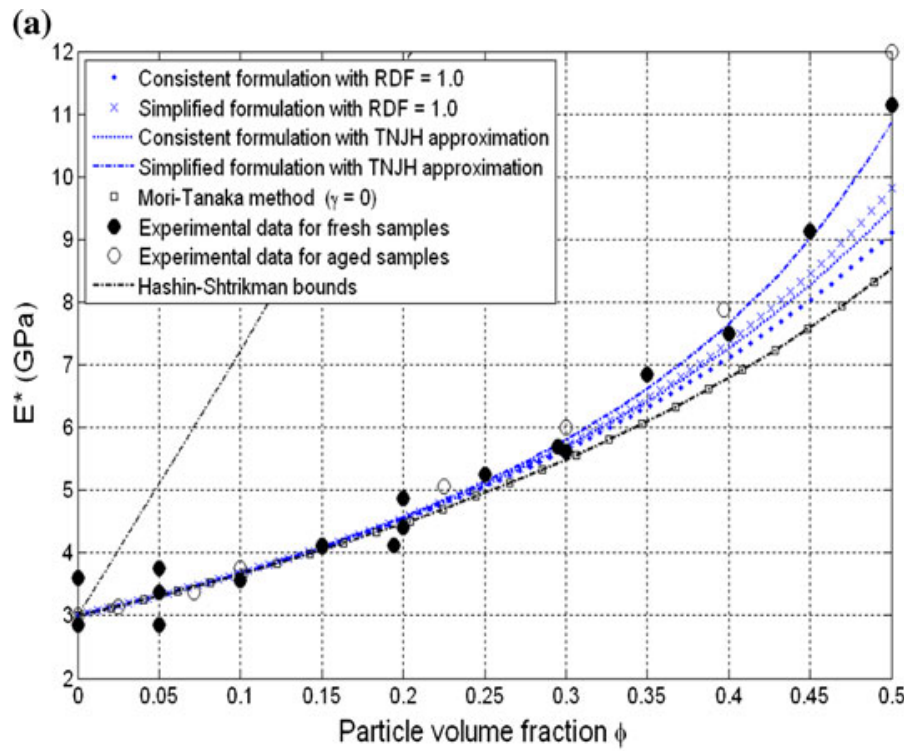

(b)

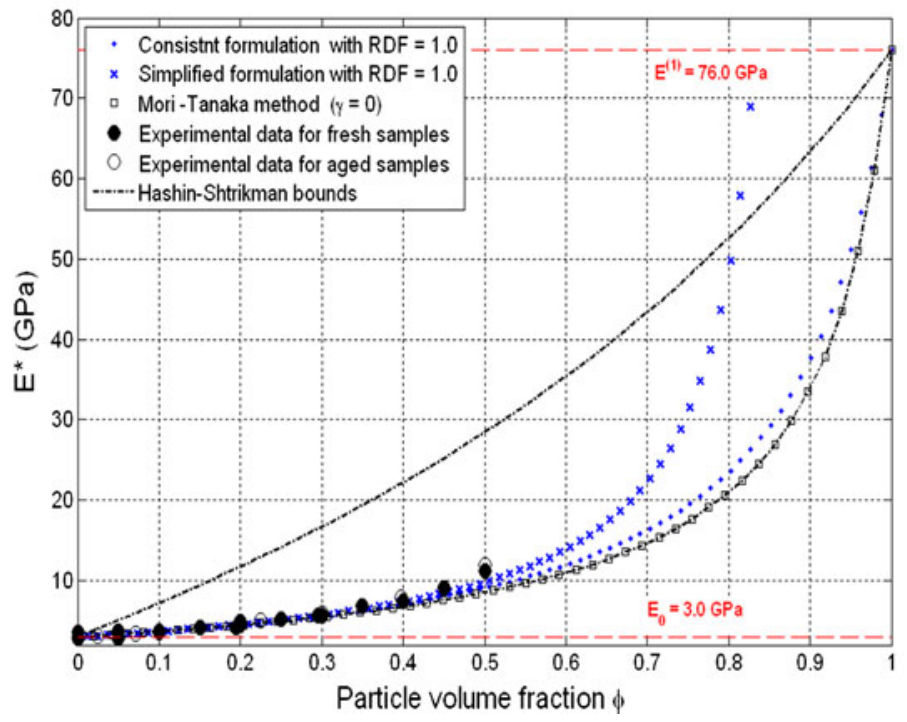

Fig. 4 The comparisons between the theoretical predictions and experimental data [27] for the effective Young's modulus $E^{*}$. a The range of volume fraction: $0 \leq \phi \leq 0.5$. b The range of volume fraction: $0 \leq \phi \leq 1.0$. Glass-Epoxy composite: $E_{0}=3.0$ $\mathrm{GPa}, v_{0}=0.4, E^{1}=76.0 \mathrm{GPa}, v^{1}=0.23$

Consequently, by using Eqs. (31), (33), (35), and (36), the effective elastic stiffness of two-phase composites can be rendered as:

$$
\begin{aligned}
& \mathbf{C}^{*}=\mathbf{C}_{0} \bullet\left[\mathbf{I}+\phi\left(\mathbf{A}^{(1)}-\overline{\mathbf{A}}^{(1)}\right)^{-1} \bullet(\mathbf{I}+\phi \mathbf{H})^{-1}\right], \\
& \mathbf{C}^{*}=\mathbf{C}_{0} \bullet\left[\mathbf{I}+\phi\left(\mathbf{A}^{(1)}-\overline{\mathbf{A}}^{(1)}\right)^{-1} \bullet(\mathbf{I}+\phi \mathbf{K})^{-1}\right], \quad \text { for consistent formulation } \\
&
\end{aligned}
$$

A specific case is considered to illustrate the intriguing feature of the proposed micromechanical formulation. By setting $\overline{\mathbf{A}}=\mathbf{0}$, one can show that:

$$
\mathbf{H}=\left(\Delta \mathbf{C}^{(1)}\right)^{-1} \bullet \mathbf{C}_{0} \bullet\left(\mathbf{A}^{(1)}\right)^{-1}-\mathbf{I}=\left[\left(\Delta \mathbf{C}^{(1)}\right)^{-1} \bullet \mathbf{C}_{0}+\mathbf{S}\right] \bullet\left[\left(\Delta \mathbf{C}^{(1)}\right)^{-1} \bullet \mathbf{C}_{0}+\mathbf{S}\right]^{-1},
$$




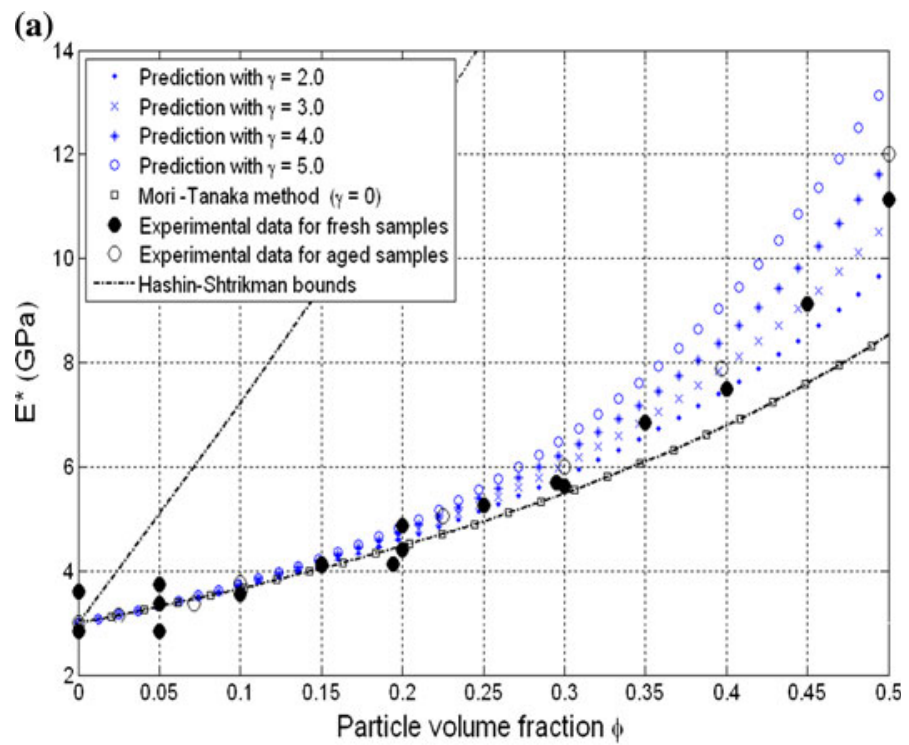

(b)

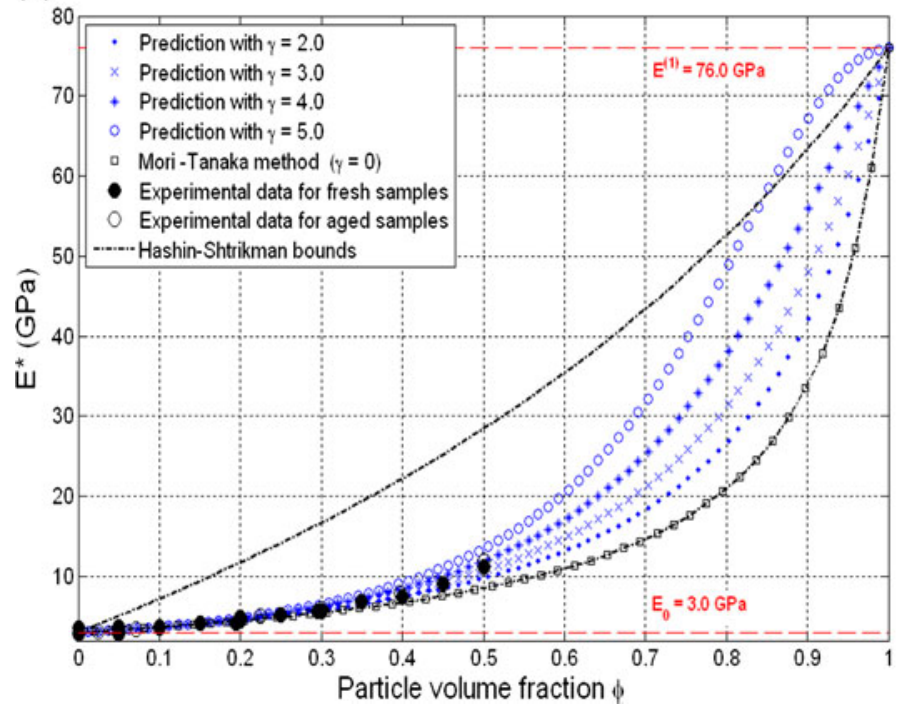

Fig. 5 The comparisons between the consistent formulation and experimental data [27] for the effective Young's modulus $E^{*}$. a The range of volume fraction: $0 \leq \phi \leq 0.5$. b The range of volume fraction: $0 \leq \phi \leq 1.0$. Glass-Epoxy composite: $E_{0}=3.0$ $\mathrm{GPa}, v_{0}=0.4, E^{1}=76.0 \mathrm{GPa}, v^{1}=0.23$

$$
-\mathbf{S} \bullet\left[\left(\Delta \mathbf{C}^{(1)}\right)^{-1} \bullet \mathbf{C}_{0}+\mathbf{S}\right]^{-1}-\mathbf{I}=-\mathbf{S} \bullet\left[\left(\Delta \mathbf{C}^{(1)}\right)^{-1} \bullet \mathbf{C}_{0}+\mathbf{S}\right]^{-1}=\mathbf{K}
$$

Accordingly, in the case of $\overline{\mathbf{A}}=\mathbf{0}$, both Eqs. (37) and (38) lead to the following expression:

$$
\mathbf{C}^{*}=\mathbf{C}_{0} \bullet\left\{\mathbf{I}+\phi\left[\Delta \mathbf{C}^{(1)} \bullet \mathbf{C}_{0}+(1-\phi) \mathbf{S}\right]^{-1}\right\}
$$

In essence, Eq. (40) is the effective elastic stiffness predicted by the Mori-Tanaka method [23,24] or the $\mathrm{Ju}-\mathrm{Chen}$ 's first-order formulation [4], in which only the far-field interactions are accounted for (cf. [2]). Obviously, the $\overline{\mathbf{A}}$ tensor in the proposed micromechanical formulation is directly related to the near-field particle interactions. 


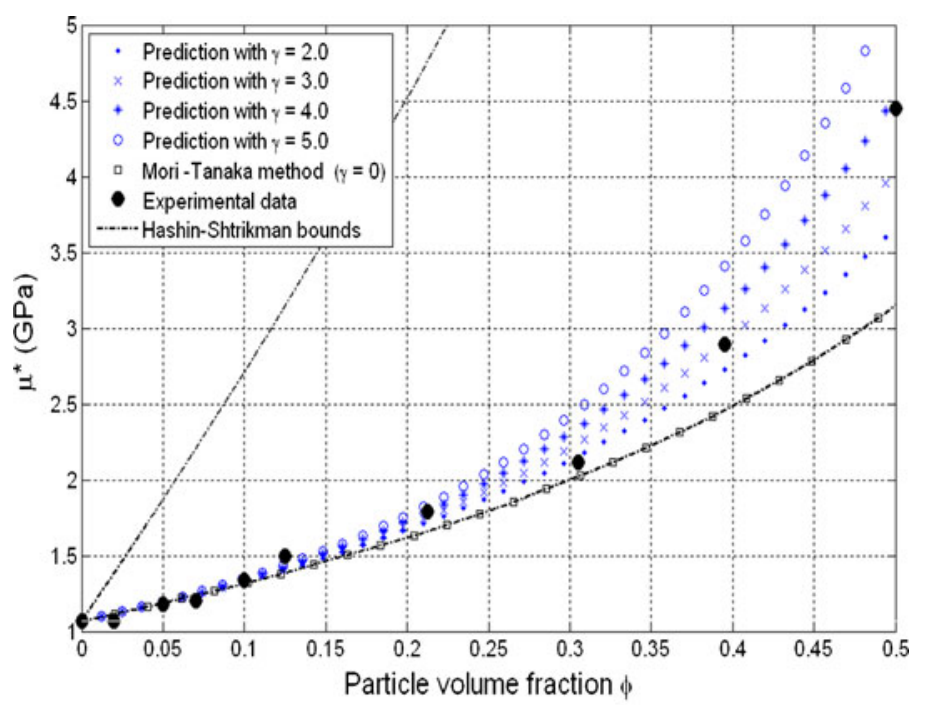

(a)

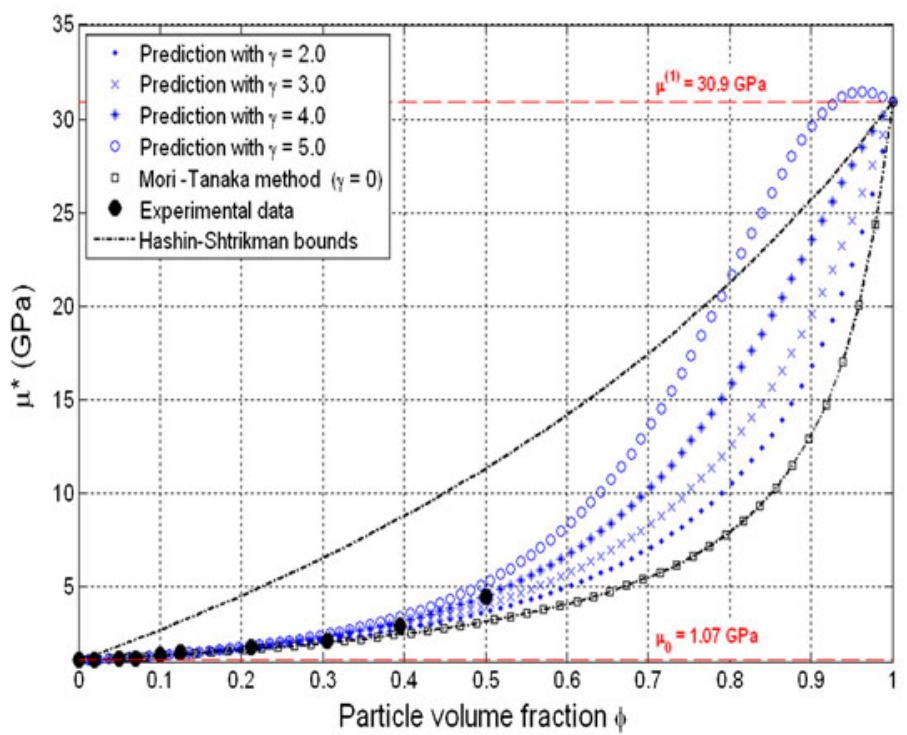

(b)

Fig. 6 The comparisons between the consistent formulation and experimental data [27] for the effective shear modulus $\mu^{*}$. a The range of volume fraction: $0 \leq \phi \leq 0.5$. b The range of volume fraction: $0 \leq \phi \leq 1.0$. Glass-Epoxy composite: $E_{0}=3.0 \mathrm{GPa}$, $n u_{0}=0.4, E^{1}=76.0 \mathrm{GPa}, v^{1}=0.23$

\section{Numerical simulations and discussions}

To demonstrate the predictive capability of the proposed micromechanical framework, comparisons are made between the theoretical predictions and the experimental data for the effective elastic moduli of two-phase composites. Further, for illustrative purposes, the predictions by the Hashin-Shtrikman bounds [25] and the Mori-Tanaka method are also provided. The Hashin-Shtrikman bounds for bulk modulus $\kappa^{*}$ and shear modulus $\mu^{*}$ are exhibited as

$$
\begin{gathered}
\kappa_{0}+\frac{\phi}{\frac{1}{\kappa^{(1)}-\kappa_{0}}+\frac{(1-\phi)}{3 \kappa_{0}+4 \mu_{0}}} \leq \kappa^{*} \leq \kappa^{(1)}+\frac{(1-\phi)}{\frac{1}{\kappa_{0}-\kappa^{(1)}}+\frac{\phi}{3 \kappa^{(1)}+4 \mu^{(1)}}} \\
\mu_{0}+\frac{\frac{1}{(1-\phi)}}{\frac{\phi}{\mu^{(1)}-\mu_{0}}+\frac{6(1-\phi)\left(\kappa_{0}+2 \mu_{0}\right)}{5 \mu_{0}\left(3 \kappa_{0}+4 \mu_{0}\right)}} \leq \mu^{*} \leq \mu^{(1)}+\frac{\frac{1}{\frac{1}{\mu_{0}-\mu^{(1)}}+\frac{6 \phi\left(\kappa^{(1)}+2 \mu^{(1)}\right)}{5 \mu^{(1)}\left(3 \kappa^{(1)}+4 \mu^{(1)}\right)}}}{}
\end{gathered}
$$


(a)

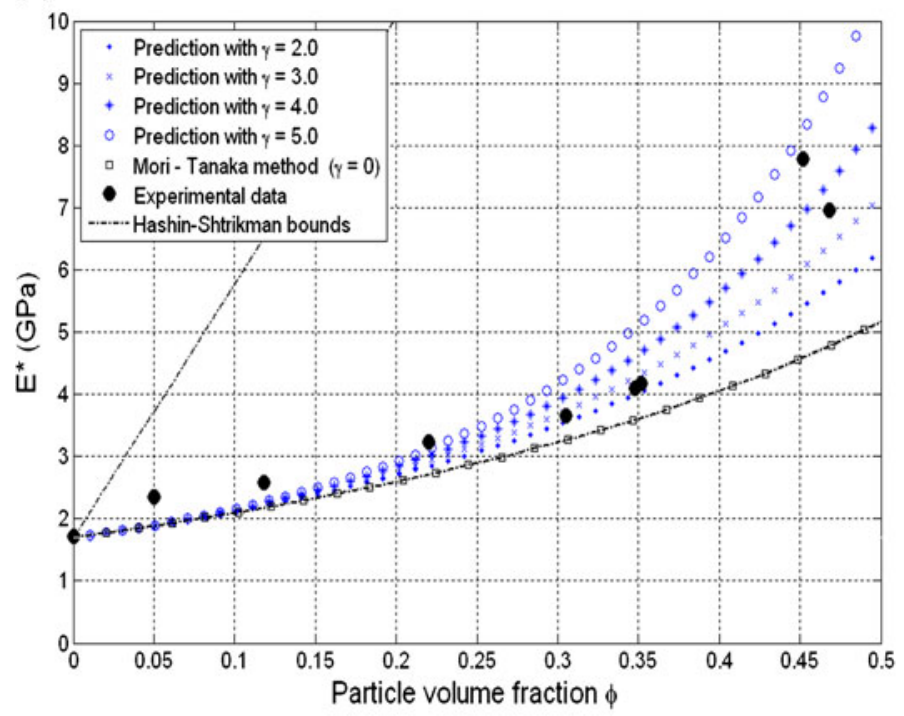

(b)

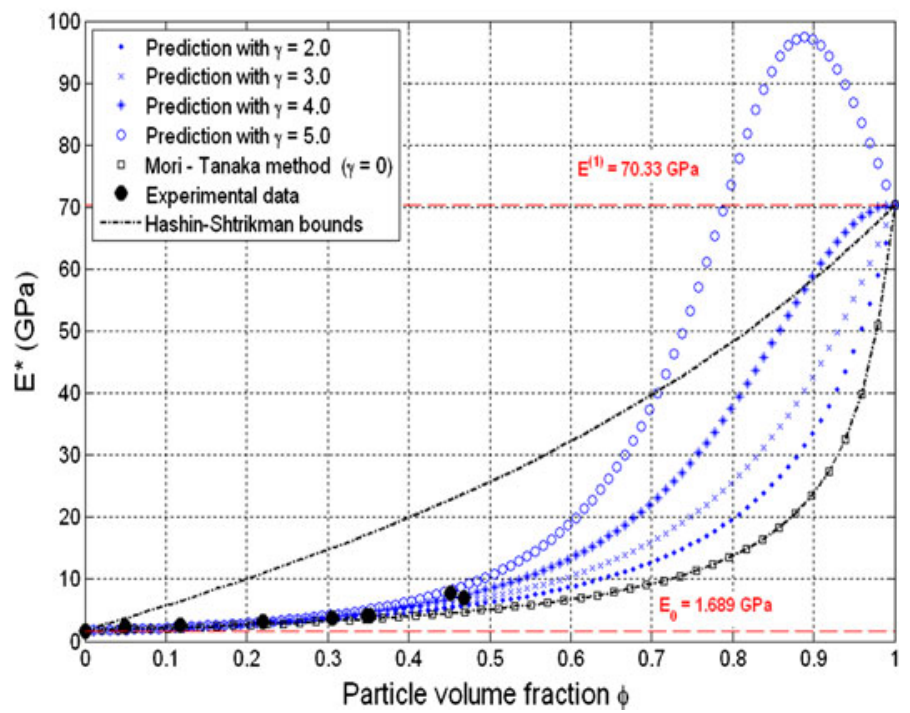

Fig. 7 The comparisons between the consistent formulation and experimental data [28] for the effective Young's modulus $E^{*}$. a The range of volume fraction: $0 \leq \phi \leq 0.5$. b The range of volume fraction: $0 \leq \phi \leq 1.0$. Glass-reinforced Polyester: $E_{0}=1.689 \mathrm{GPa}, v_{0}=0.444, E^{1}=70.33 \mathrm{GPa}, v^{1}=0.21$

As illustrated in Fig. 4a, the simplified formulation can reproduce the experimental data with better accuracy than the consistent formulation. Moreover, the introduction of the radial distribution function improves the predictive capability. Since the TNJH approximation cannot be used for $\phi>0.5$, the uniform radial distribution $(g(r)=1)$ is adopted in Fig. 4b. Despite its good predictive capability in Fig. 4a, the simplified formulation hits a singularity and predicts an infinite elastic modulus at very high particle concentration, thus violating the mathematical upper bound. By contrast, the predictions with the consistent formulation are always bounded within the mathematical bounds, reproducing the modulus of the particle at $\phi=1.0$. It is noted that the Hashin-Shtrikman lower bound and the Mori-Tanaka method render identical predictions for the effective moduli of composites (cf. [4,24,26]).

Once $g(r)$ is involved, the integration of Eq. (29) becomes quite tedious, leading to a significant increase in computation time. Nevertheless, the incorporation of $g(r)$ can slightly improve the theoretical predictions, in particular, for the consistent formulation. To overcome these difficulties, the simplest approach that we propose is to approximate Eq. (29) by the following expression: 


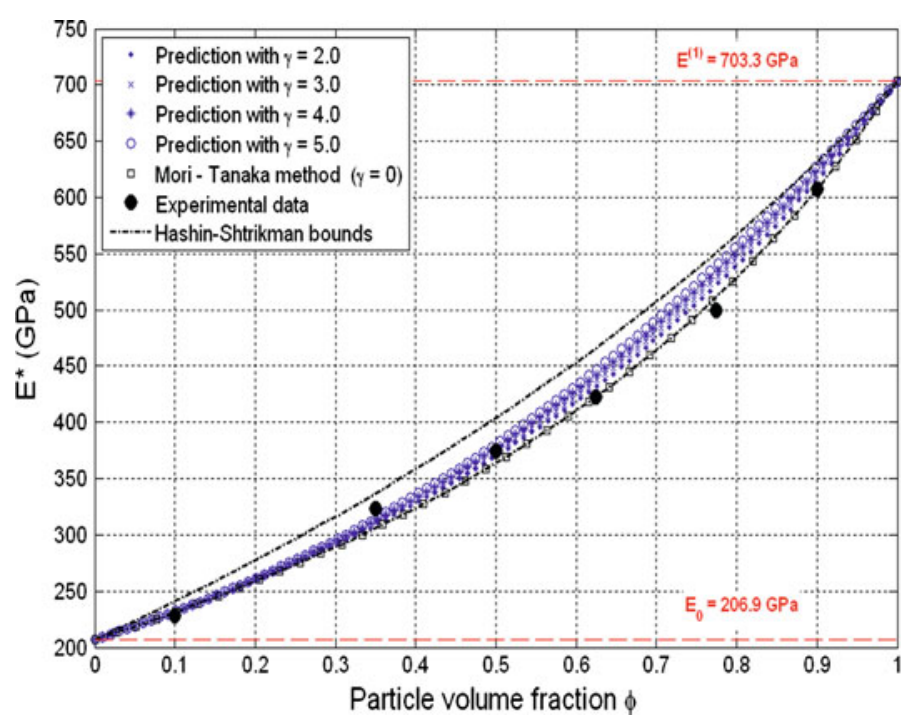

Fig. 8 The comparisons between the consistent formulation and experimental data [29] for the effective Young's modulus $E^{*}$. $W_{\mathrm{c}}-$ Co composite: $E_{o}=206.9 \mathrm{GPa}, v_{0}=0.304, E^{(1)}=703.3 \mathrm{GPa}, v^{(1)}=0.22$

$$
\overline{\mathbf{A}}^{(r)}=\frac{N^{(r)}}{V} \int_{V \notin \Omega^{(r)}} \hat{\mathbf{G}} \bullet\left(\mathbf{A}^{(r)}\right)^{-1} \bullet \hat{\mathbf{G}} \cdot g(r) \mathrm{d} V \approx \frac{N^{(r)}}{V} \gamma \int_{V \notin \Omega^{(r)}} \hat{\mathbf{G}} \bullet\left(\mathbf{A}^{(r)}\right)^{-1} \bullet \hat{\mathbf{G}} \mathrm{d} V .
$$

Here, it is assumed that a parameter $\gamma$ represents the overall effect of $g(r)$ within an $R V E$. In reality, $\gamma$ is not a constant, it should be a function of $\phi$ at least. It is noted that, due to the approximation, Eq. (43) may not truly reflect the microstructural effects. However, we still consider the effects of particle locations with approximation in Eq. (43). Hence, the proposed formulation may be regarded as a mostly micromechanical formulation. In what follows, to further investigate the proposed micromechanical framework, parametric studies on the parameter $\gamma$ are performed with the consistent formulation. By using Eq. (43) with $\gamma=$ constant, it is shown that we can reproduce various experimental data with good accuracy.

In Fig. 5, the effective Young's moduli are predicted with different values of $\gamma$. As observed, when $\gamma \cong 3.5$, the predictions are in good agreement with the experimental data. However, when $\gamma=5$, the consistent predictions overestimate the effective modulus, and violate the mathematical upper bound around $\phi \cong 0.85$. Similar phenomena are observed in Figs. 6 and 7. In these figures, both the mathematical upper bounds and the physical upper bounds $\left(\mu^{(1)}, E^{(1)}\right)$ are violated when $\gamma=5$. From these observations, it is clear that a large value for $\gamma$ cannot be employed in the proposed micromechanical formulation. Moreover, according to our rough estimation, Ju and Chen's lower and upper bounds [5] correspond to $\gamma \approx 3.5$ and $\gamma \approx 4.5$, respectively.

Figure 8 exhibits the predictions on the effective Young's modulus. Interestingly, variations in $\gamma$ do not lead to significant changes in the predictions. Instead, they are well within the narrow band of mathematical bounds. The results from Fig. 8 do not suggest that the near-field interactions are not important. Alternatively, it exemplifies that unique combinations of matrix and reinforcing-phase properties result in the unique intensities of near-field interactions. Finally, Fig. 9 displays the effective bulk modulus of porous media (glass). In comparison with the Mori-Tanaka method, the consistent formulation with the parameter $\gamma$ can reproduce the experimental data with better accuracy.

In reality, the size of particles has a characteristic size distribution. In addition, the interactions are among many particles instead of pairwise. Therefore, an appropriate particle-size distribution function and an approximate yet reasonable multi-particle interaction solution may enhance the predictive capability of the proposed micromechanical framework without resorting to the parameter $\gamma$.

\section{Conclusions}

By taking advantage of the probabilistic particle interacting solution within the micromechanical framework, the higher-order (in terms of $\phi$ ) micromechanical formulation is proposed to predict the effective elastic moduli of multi-phase composites containing randomly dispersed spherical particles. As a special case, the effective 

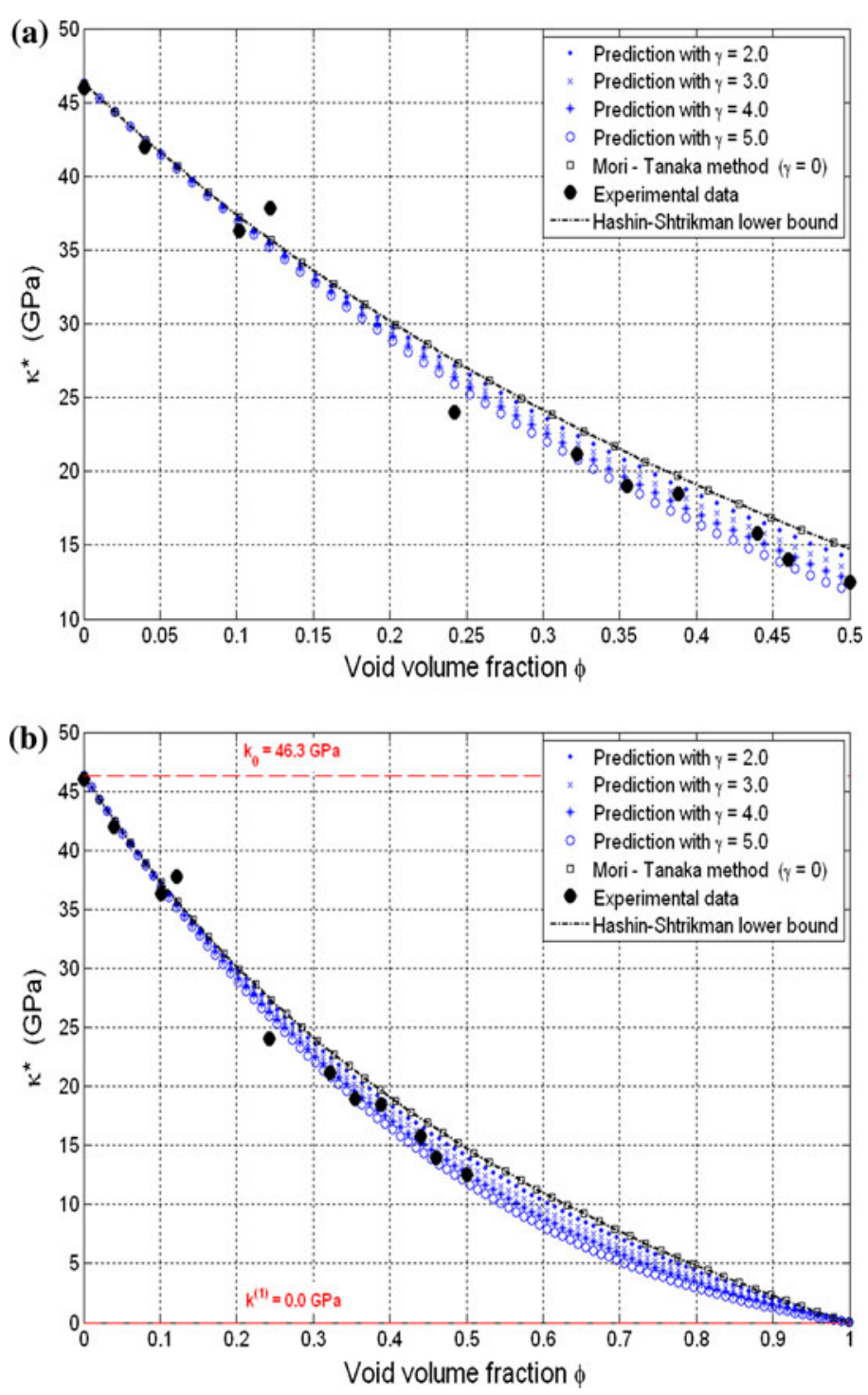

Fig. 9 The comparisons between the consistent formulation and experimental data [30] for the effective bulk modulus $\kappa^{*}$ of porous media. a The range of volume fraction: $0 \leq \phi \leq 0.5$. b The range of volume fraction: $0 \leq \phi \leq 1.0$. $E_{0}=75 \mathrm{GPa}$, $v_{0}=0.23$

elastic moduli of two-phase composites containing the isotropic spherical particles are predicted. By using the parameter $\gamma$, the micromechanical predictions can reproduce available experimental data with good accuracy under reasonable $\gamma$ values. The proposed analytical framework can be readily extended to accommodate anisotropic particles and to account for particles of different sizes or shapes with added effort and complexity.

Acknowledgments This work is in part sponsored by the Faculty Research Grant of the Academic Senate of UCLA (Fund Number 4-592565-19914) and in part by the Bellagio Engineering.

Open Access This article is distributed under the terms of the Creative Commons Attribution Noncommercial License which permits any noncommercial use, distribution, and reproduction in any medium, provided the original author(s) and source are credited.

\section{Appendix I}

Let us define the following equation: 


$$
\overline{\mathbf{A}}^{(r)}=\frac{N^{(r)}}{V} \int_{V \notin \Omega^{(r)}} \hat{\mathbf{G}} \bullet\left(\mathbf{A}^{(r)}\right)^{-1} \bullet \hat{\mathbf{G}} \mathrm{d} V=\frac{N^{(r)}}{V} \int_{V \notin \Omega^{(r)}} \hat{\mathbf{G}} \bullet \mathbf{L}^{(r)} \bullet \hat{\mathbf{G}} \mathrm{d} V .
$$

The tensorial components of Eq. (44) take the forms:

$$
\begin{aligned}
& \frac{\bar{A}_{1111}^{(r)}}{\phi^{(r)}}=L_{1111}^{(r)} \frac{51-80 v_{0}+35 v_{0}^{2}}{3150\left(1-v_{0}\right)^{2}}+L_{1122}^{(r)} \frac{-11-35 v_{0}+35 v_{0}^{2}}{6300\left(1-v_{0}\right)^{2}}+L_{1133}^{(r)} \frac{-11-35 v_{0}+35 v_{0}^{2}}{6300\left(1-v_{0}\right)^{2}} \\
& +L_{2211}^{(r)} \frac{-11+85 v_{0}-70 v_{0}^{2}}{6300\left(1-v_{0}\right)^{2}}+L_{2222}^{(r)} \frac{59-80 v_{0}-280 v_{0}^{2}}{50400\left(1-v_{0}\right)^{2}}+L_{2233}^{(r)} \frac{-11+40 v_{0}-280 v_{0}^{2}}{50400(1-v)^{2}} \\
& +L_{3311}^{(r)} \frac{-11+85 v_{0}-70 v_{0}^{2}}{6300\left(1-v_{0}\right)^{2}}+L_{3322}^{(r)} \frac{-11+40 v_{0}-280 v_{0}^{2}}{50400\left(1-v_{0}\right)^{2}}+L_{3333}^{(r)} \frac{59-80 v_{0}-280 v_{0}^{2}}{50400\left(1-v_{0}\right)^{2}} \\
& +L_{2323}^{(r)} \frac{7-12 v_{0}}{2520\left(1-v_{0}\right)^{2}}+L_{1313}^{(r)} \frac{19-24 v_{0}}{1260\left(1-v_{0}\right)^{2}}+L_{1212}^{(r)} \frac{19-24 v_{0}}{1260\left(1-v_{0}\right)^{2}}, \\
& \frac{\bar{A}_{1122}^{(r)}}{\phi^{(r)}}=L_{1111}^{(r)} \frac{-11+85 v_{0}-70 v_{0}^{2}}{6300\left(1-v_{0}\right)^{2}}+L_{1122}^{(r)} \frac{-167+320 v_{0}-140 v_{0}^{2}}{25200\left(1-v_{0}\right)^{2}}+L_{1133}^{(r)} \frac{51-200 v_{0}+140 v_{0}^{2}}{25200\left(1-v_{0}\right)^{2}} \\
& +L_{2211}^{(r)} \frac{59-80 v_{0}+560 v_{0}^{2}}{50400\left(1-v_{0}\right)^{2}}+L_{2222}^{(r)} \frac{-11-35 v_{0}+35 v_{0}^{2}}{6300\left(1-v_{0}\right)^{2}}+L_{2233}^{(r)} \frac{-11+160 v_{0}-280 v_{0}^{2}}{50400\left(1-v_{0}\right)^{2}} \\
& +L_{3311}^{(r)} \frac{-11-80 v_{0}+560 v_{0}^{2}}{50400\left(1-v_{0}\right)^{2}}+L_{3322}^{(r)} \frac{51-200 v_{0}+140 v_{0}^{2}}{25200\left(1-v_{0}\right)^{2}}+L_{3333}^{(r)} \frac{-11+40 v_{0}-280 v_{0}^{2}}{50400\left(1-v_{0}\right)^{2}} \\
& +L_{2323}^{(r)} \frac{-19}{5040\left(1-v_{0}\right)^{2}}+L_{1313}^{(r)} \frac{-19+120 v_{0}-168 v_{0}^{2}}{5040\left(1-v_{0}\right)^{2}}+L_{1212}^{(r)} \frac{13-32 v_{0}}{1680\left(1-v_{0}\right)^{2}} \\
& \frac{\bar{A}_{1133}^{(r)}}{\phi^{(r)}}=L_{1111}^{(r)} \frac{-11+85 v_{0}-70 v_{0}^{2}}{6300\left(1-v_{0}\right)^{2}}+L_{1122}^{(r)} \frac{51-200 v_{0}+140 v_{0}^{2}}{25200\left(1-v_{0}\right)^{2}}+L_{1133}^{(r)} \frac{-167+320 v_{0}-140 v_{0}^{2}}{25200\left(1-v_{0}\right)^{2}} \\
& +L_{2211}^{(r)} \frac{-11-80 v_{0}+560 v_{0}^{2}}{50400\left(1-v_{0}\right)^{2}}+L_{2222}^{(r)} \frac{-11+40 v_{0}-280 v_{0}^{2}}{50400\left(1-v_{0}\right)^{2}}+L_{2233}^{(r)} \frac{51-200 v_{0}+140 v_{0}^{2}}{25200\left(1-v_{0}\right)^{2}} \\
& +L_{3311}^{(r)} \frac{59-80 v_{0}+560 v_{0}^{2}}{50400\left(1-v_{0}\right)^{2}}+L_{3322}^{(r)} \frac{-11+160 v_{0}-280 v_{0}^{2}}{50400\left(1-v_{0}\right)^{2}}+L_{3333}^{(r)} \frac{-11-35 v_{0}+35 v_{0}^{2}}{6300\left(1-v_{0}\right)^{2}} \\
& +L_{2323}^{(r)} \frac{-19}{5040\left(1-v_{0}\right)^{2}}+L_{1313}^{(r)} \frac{13-32 v_{0}}{1680\left(1-v_{0}\right)^{2}}+L_{1212}^{(r)} \frac{-19+120 v_{0}-168 v_{0}^{2}}{5040\left(1-v_{0}\right)^{2}} \\
& \frac{\bar{A}_{2211}^{(r)}}{\phi^{(r)}}=L_{1111}^{(r)} \frac{-11-35 v_{0}+35 v_{0}^{2}}{6300\left(1-v_{0}\right)^{2}}+L_{1122}^{(r)} \frac{59-80 v_{0}+560 v_{0}^{2}}{50400\left(1-v_{0}\right)^{2}}+L_{1133}^{(r)} \frac{-11+160 v_{0}-280 v_{0}^{2}}{50400\left(1-v_{0}\right)^{2}} \\
& +L_{2211}^{(r)} \frac{-167+320 v_{0}-140 v_{0}^{2}}{25200\left(1-v_{0}\right)^{2}}+L_{2222}^{(r)} \frac{-11+85 v_{0}-70 v_{0}^{2}}{6300\left(1-v_{0}\right)^{2}}+L_{2233}^{(r)} \frac{51-200 v_{0}+140 v_{0}^{2}}{25200\left(1-v_{0}\right)^{2}} \\
& +L_{3311}^{(r)} \frac{51-200 v_{0}+140 v_{0}^{2}}{25200\left(1-v_{0}\right)^{2}}+L_{3322}^{(r)} \frac{-11-80 v_{0}+560 v_{0}^{2}}{50400\left(1-v_{0}\right)^{2}}+L_{3333}^{(r)} \frac{-11+40 v_{0}-280 v_{0}^{2}}{50400\left(1-v_{0}\right)^{2}} \\
& +L_{2323}^{(r)} \frac{-19+120 v_{0}-168 v_{0}^{2}}{5040\left(1-v_{0}\right)^{2}}+L_{1313}^{(r)} \frac{-19}{5040\left(1-v_{0}\right)^{2}}+L_{1212}^{(r)} \frac{13-32 v_{0}}{1680\left(1-v_{0}\right)^{2}}, \\
& \frac{\bar{A}_{2222}^{(r)}}{\phi^{(r)}}=L_{1111}^{(r)} \frac{59-80 v_{0}-28 v_{0}^{2}}{50400\left(1-v_{0}\right)^{2}}+L_{1122}^{(r)} \frac{-11+85 v_{0}-70 v_{0}^{2}}{6300\left(1-v_{0}\right)^{2}}+L_{1133}^{(r)} \frac{-11+40 v_{0}-280 v_{0}^{2}}{50400\left(1-v_{0}\right)^{2}} \\
& +L_{2211}^{(r)} \frac{-11-35 v_{0}+35 v_{0}^{2}}{6300\left(1-v_{0}\right)^{2}}+L_{2222}^{(r)} \frac{51-80 v_{0}+35 v_{0}^{2}}{3150\left(1-v_{0}\right)^{2}}+L_{2233}^{(r)} \frac{-11-35 v_{0}+35 v_{0}^{2}}{6300\left(1-v_{0}\right)^{2}}
\end{aligned}
$$




$$
\begin{aligned}
& +L_{3311}^{(r)} \frac{-11+40 v_{0}-280 v_{0}^{2}}{50400\left(1-v_{0}\right)^{2}}+L_{3322}^{(r)} \frac{-11+85 v_{0}-70 v_{0}^{2}}{6300\left(1-v_{0}\right)^{2}}+L_{3333}^{(r)} \frac{59-80 v_{0}-280 v_{0}^{2}}{50400\left(1-v_{0}\right)^{2}} \\
& +L_{2323}^{(r)} \frac{19-24 v_{0}}{1260\left(1-v_{0}\right)^{2}}+L_{1313}^{(r)} \frac{7-12 v_{0}}{2520\left(1-v_{0}\right)^{2}}+L_{1212}^{(r)} \frac{19-24 v_{0}}{1260\left(1-v_{0}\right)^{2}}, \\
& \frac{\bar{A}_{2233}^{(r)}}{\phi^{(r)}}=L_{1111}^{(r)} \frac{-11+40 v_{0}-280 v_{0}^{2}}{50400\left(1-v_{0}\right)^{2}}+L_{1122}^{(r)} \frac{-11-80 v_{0}+560 v_{0}^{2}}{50400\left(1-v_{0}\right)^{2}}+L_{1133}^{(r)} \frac{51-200 v_{0}+140 v_{0}^{2}}{25200\left(1-v_{0}\right)^{2}} \\
& +L_{2211}^{(r)} \frac{51-200 v_{0}+140 v_{0}^{2}}{25200\left(1-v_{0}\right)^{2}}+L_{2222}^{(r)} \frac{-11+85 v_{0}-70 v_{0}^{2}}{6300\left(1-v_{0}\right)^{2}}+L_{2233}^{(r)} \frac{-167+320 v_{0}-140 v_{0}^{2}}{25200\left(1-v_{0}\right)^{2}} \\
& +L_{3311}^{(r)} \frac{-11+160 v_{0}-280 v_{0}^{2}}{50400\left(1-v_{0}\right)^{2}}+L_{3322}^{(r)} \frac{59-80 v_{0}+560 v_{0}^{2}}{50400\left(1-v_{0}\right)^{2}}+L_{3333}^{(r)} \frac{-11-35 v_{0}+35 v_{0}^{2}}{6300\left(1-v_{0}\right)^{2}} \\
& +L_{2323}^{(r)} \frac{13-32 v_{0}}{1680\left(1-v_{0}\right)^{2}}+L_{1313}^{(r)} \frac{-19}{5040\left(1-v_{0}\right)^{2}}+L_{1212}^{(r)} \frac{-19+120 v_{0}-168 v_{0}^{2}}{5040\left(1-v_{0}\right)^{2}} \\
& \frac{\bar{A}_{3311}^{(r)}}{\phi^{(r)}}=L_{1111}^{(r)} \frac{-11-35 v_{0}+35 v_{0}^{2}}{6300\left(1-v_{0}\right)^{2}}+L_{1122}^{(r)} \frac{-11+160 v_{0}-280 v_{0}^{2}}{50400\left(1-v_{0}\right)^{2}}+L_{1133}^{(r)} \frac{59-80 v_{0}+560 v_{0}^{2}}{50400\left(1-v_{0}\right)^{2}} \\
& +L_{2211}^{(r)} \frac{51-200 v_{0}+140 v_{0}^{2}}{25200\left(1-v_{0}\right)^{2}}+L_{2222}^{(r)} \frac{-11+40 v_{0}-280 v_{0}^{2}}{50400\left(1-v_{0}\right)^{2}}+L_{2233}^{(r)} \frac{-11-80 v_{0}+560 v_{0}^{2}}{50400\left(1-v_{0}\right)^{2}} \\
& +L_{3311}^{(r)} \frac{-167+320 v_{0}-140 v_{0}^{2}}{25200\left(1-v_{0}\right)^{2}}+L_{3322}^{(r)} \frac{51-200 v_{0}+140 v_{0}^{2}}{25200\left(1-v_{0}\right)^{2}}+L_{3333}^{(r)} \frac{-11+85 v_{0}-70 v_{0}^{2}}{6300\left(1-v_{0}\right)^{2}} \\
& +L_{2323}^{(r)} \frac{-19+120 v_{0}-168 v_{0}^{2}}{5040\left(1-v_{0}\right)^{2}}+L_{1313}^{(r)} \frac{13-32 v_{0}}{1680\left(1-v_{0}\right)^{2}}+L_{1212}^{(r)} \frac{-19}{5040\left(1-v_{0}\right)^{2}} \\
& \frac{\bar{A}_{3322}^{(r)}}{\phi^{(r)}}=L_{1111}^{(r)} \frac{-11+40 v_{0}-280 v_{0}^{2}}{50400\left(1-v_{0}\right)^{2}}+L_{1122}^{(r)} \frac{51-200 v_{0}+140 v_{0}^{2}}{25200\left(1-v_{0}\right)^{2}}+L_{1133}^{(r)} \frac{-11-80 v_{0}+560 v_{0}^{2}}{50400\left(1-v_{0}\right)^{2}} \\
& +L_{2211}^{(r)} \frac{-11+160 v_{0}-280 v_{0}^{2}}{50400\left(1-v_{0}\right)^{2}}+L_{2222}^{(r)} \frac{-11-35 v_{0}+35 v_{0}^{2}}{6300\left(1-v_{0}\right)^{2}}+L_{2233}^{(r)} \frac{59-80 v_{0}+560 v_{0}^{2}}{50400\left(1-v_{0}\right)^{2}} \\
& +L_{3311}^{(r)} \frac{51-200 v_{0}+140 v_{0}^{2}}{25200\left(1-v_{0}\right)^{2}}+L_{3322}^{(r)} \frac{-167+320 v_{0}-140 v_{0}^{2}}{25200\left(1-v_{0}\right)^{2}}+L_{3333}^{(r)} \frac{-11+85 v_{0}-70 v_{0}^{2}}{6300\left(1-v_{0}\right)^{2}} \\
& +L_{2323}^{(r)} \frac{13-32 v_{0}}{1680\left(1-v_{0}\right)^{2}}+L_{1313}^{(r)} \frac{-19+120 v_{0}-168 v_{0}^{2}}{5040\left(1-v_{0}\right)^{2}}+L_{1212}^{(r)} \frac{-19}{5040\left(1-v_{0}\right)^{2}}, \\
& \frac{\bar{A}_{3333}^{(r)}}{\phi^{(r)}}=L_{1111}^{(r)} \frac{59-80 v_{0}-280 v_{0}^{2}}{50400\left(1-v_{0}\right)^{2}}+L_{1122}^{(r)} \frac{-11+40 v_{0}-280 v_{0}^{2}}{50400\left(1-v_{0}\right)^{2}}+L_{1133}^{(r)} \frac{-11+85 v_{0}-70 v_{0}^{2}}{6300\left(1-v_{0}\right)^{2}} \\
& +L_{2211}^{(r)} \frac{-11+40 v_{0}-280 v_{0}^{2}}{50400\left(1-v_{0}\right)^{2}}+L_{2222}^{(r)} \frac{59-80 v_{0}-280 v_{0}^{2}}{50400\left(1-v_{0}\right)^{2}}+L_{2233}^{(r)} \frac{-11+85 v_{0}-70 v_{0}^{2}}{6300\left(1-v_{0}\right)^{2}} \\
& +L_{3311}^{(r)} \frac{-11-35 v_{0}+35 v_{0}^{2}}{6300\left(1-v_{0}\right)^{2}}+L_{3322}^{(r)} \frac{-11-35 v_{0}+35 v_{0}^{2}}{6300\left(1-v_{0}\right)^{2}}+L_{3333}^{(r)} \frac{51-80 v_{0}+35 v_{0}^{2}}{3150\left(1-v_{0}\right)^{2}} \\
& +L_{2323}^{(r)} \frac{19-24 v_{0}}{1260\left(1-v_{0}\right)^{2}}+L_{1313}^{(r)} \frac{19-24 v_{0}}{1260\left(1-v_{0}\right)^{2}}+L_{1212}^{(r)} \frac{7-12 v_{0}}{2520\left(1-v_{0}\right)^{2}}, \\
& \frac{\bar{A}_{2323}^{(r)}}{\phi^{(r)}}=L_{1111}^{(r)} \frac{7-12 v_{0}}{10080\left(1-v_{0}\right)^{2}}+L_{1122}^{(r)} \frac{-19+120 v_{0}-168 v_{0}^{2}}{20160\left(1-v_{0}\right)^{2}}+L_{1133}^{(r)} \frac{-19+120 v_{0}-168 v_{0}^{2}}{20160\left(1-v_{0}\right)^{2}} \\
& +L_{2211}^{(r)} \frac{-19}{20160\left(1-v_{0}\right)^{2}}+L_{2222}^{(r)} \frac{19-24 v_{0}}{5040\left(1-v_{0}\right)^{2}}+L_{2233}^{(r)} \frac{13-32 v_{0}}{6720\left(1-v_{0}\right)^{2}}
\end{aligned}
$$




$$
\begin{aligned}
& +L_{3311}^{(r)} \frac{-19}{20160\left(1-v_{0}\right)^{2}}+L_{3322}^{(r)} \frac{13-32 v_{0}}{6720\left(1-v_{0}\right)^{2}}+L_{3333}^{(r)} \frac{19-24 v_{0}}{5040\left(1-v_{0}\right)^{2}} \\
& +L_{2323}^{(r)} \frac{119-200 v_{0}+140 v_{0}^{2}}{12600\left(1-v_{0}\right)^{2}}+L_{1313}^{(r)} \frac{16-30 v_{0}+21 v_{0}^{2}}{2520\left(1-v_{0}\right)^{2}}+L_{1212}^{(r)} \frac{16-30 v_{0}+21 v_{0}^{2}}{2520\left(1-v_{0}\right)^{2}}
\end{aligned}
$$

$$
\begin{aligned}
\frac{\bar{A}_{1313}^{(r)}}{\phi^{(r)}}= & L_{1111}^{(r)} \frac{19-24 v_{0}}{5040\left(1-v_{0}\right)^{2}}+L_{1122}^{(r)} \frac{-19}{20160\left(1-v_{0}\right)^{2}}+L_{1133}^{(r)} \frac{13-32 v_{0}}{6720\left(1-v_{0}\right)^{2}} \\
& +L_{2211}^{(r)} \frac{-19+120 v_{0}-168 v_{0}^{2}}{20160\left(1-v_{0}\right)^{2}}+L_{2222}^{(r)} \frac{7-12 v_{0}}{10080\left(1-v_{0}\right)^{2}}+L_{2233}^{(r)} \frac{-19+120 v_{0}-168 v_{0}^{2}}{20160\left(1-v_{0}\right)^{2}} \\
& +L_{3311}^{(r)} \frac{13-32 v_{0}}{6720\left(1-v_{0}\right)^{2}}+L_{3322}^{(r)} \frac{-19}{20160\left(1-v_{0}\right)^{2}}+L_{3333}^{(r)} \frac{19-24 v_{0}}{5040\left(1-v_{0}\right)^{2}} \\
& +L_{2323}^{(r)} \frac{16-30 v_{0}+21 v_{0}^{2}}{2520\left(1-v_{0}\right)^{2}}+L_{1313}^{(r)} \frac{119-200 v_{0}+140 v_{0}^{2}}{12600\left(1-v_{0}\right)^{2}}+L_{1212}^{(r)} \frac{16-30 v_{0}+21 v_{0}^{2}}{2520\left(1-v_{0}\right)^{2}}
\end{aligned}
$$

$$
\begin{aligned}
\frac{\bar{A}_{1212}^{(r)}}{\phi^{(r)}}= & L_{1111}^{(r)} \frac{19-24 v_{0}}{5040\left(1-v_{0}\right)^{2}}+L_{1122}^{(r)} \frac{13-32 v_{0}}{6720\left(1-v_{0}\right)^{2}}+L_{1133}^{(r)} \frac{-19}{20160\left(1-v_{0}\right)^{2}} \\
& +L_{2211}^{(r)} \frac{13-32 v_{0}}{6720\left(1-v_{0}\right)^{2}}+L_{2222}^{(r)} \frac{19-24 v_{0}}{5040\left(1-v_{0}\right)^{2}}+L_{2233}^{(r)} \frac{-19}{20160\left(1-v_{0}\right)^{2}} \\
& +L_{3311}^{(r)} \frac{-19+120 v_{0}-168 v_{0}^{2}}{20160\left(1-v_{0}\right)^{2}}+L_{3322}^{(r)} \frac{-19+120 v_{0}-168 v_{0}^{2}}{20160\left(1-v_{0}\right)^{2}}+L_{3333}^{(r)} \frac{7-12 v_{0}}{10080\left(1-v_{0}\right)^{2}} \\
& +L_{2323}^{(r)} \frac{16-30 v_{0}+21 v_{0}^{2}}{2520\left(1-v_{0}\right)^{2}}+L_{1313}^{(r)} \frac{16-30 v_{0}+21 v_{0}^{2}}{2520\left(1-v_{0}\right)^{2}}+L_{1212}^{(r)} \frac{119-200 v_{0}+140 v_{0}^{2}}{12600\left(1-v_{0}\right)^{2}}
\end{aligned}
$$

where $v_{0}$ is Poisson's ratio of the matrix material.

\section{Appendix II}

In the TNJH approximation [22], the RDF is written as:

$$
g(r)= \begin{cases}g^{d e p}(r), & \text { for } 2 a \leq r \leq r^{*} \\ g^{s t r}(r), & \text { for } r^{*} \leq r \leq \infty\end{cases}
$$

where the "depletion" (d) and "structural" (s) parts have the following forms:

$$
\begin{aligned}
g^{d e p}(r) & =\frac{A}{r} e^{\mu(r-2 a)}+\frac{B}{r} \cos (\beta[r-2 a]+\gamma) \cdot e^{\alpha(r-2 a)}, \\
g^{s t r}(r) & =1+\frac{C}{r} \cos (\omega r+\delta) \cdot e^{-\kappa r} .
\end{aligned}
$$

It is noted that $r^{*}$ is the position for the first minimum of $g(r)$, which reads:

$$
r^{*}=2 a\left(2.0116-1.0647 \phi+0.0538 \phi^{2}\right) \text {. }
$$

Moreover, the following equations need to be satisfied at $r=r^{*}$ :

$$
\begin{aligned}
g^{d e p}\left(r^{*}\right) & =g^{s t r}\left(r^{*}\right), \\
\left.\frac{\mathrm{d}}{\mathrm{d} r} g^{\operatorname{dep}}(r)\right|_{r=r^{*}} & =\left.\frac{\mathrm{d}}{\mathrm{d} r} g^{s t r}(r)\right|_{r=r^{*}} .
\end{aligned}
$$


The coefficients in Eqs. (58) and (59) are given as:

$$
\begin{aligned}
A & =2 a \cdot g_{\sigma}^{\text {expt }}-B \cos \gamma \\
B & =\frac{g_{m}-\left(2 a \cdot g_{\sigma}^{\text {expt }} / r^{*}\right) \cdot e^{\mu\left(r^{*}-2 a\right)}}{\cos \left(\beta\left[r^{*}-2 a\right]+\gamma\right) \cdot e^{\alpha\left(r^{*}-2 a\right)}-\cos \gamma \cdot e^{\mu\left(r^{*}-2 a\right)}} r^{*}, \\
C & =\frac{r^{*}\left(g_{m}-1\right) \cdot e^{\kappa r^{*}}}{\cos \left(\omega r^{*}+\delta\right)}, \\
\delta & =-\omega r^{*}-\arctan \frac{\kappa r^{*}+1}{\omega r^{*}},
\end{aligned}
$$

where

$$
\begin{aligned}
g_{m} & =1.0286-0.6095 \phi+3.7581 \phi^{2}-21.3651 \phi^{3}+42.6344 \phi^{4}-33.8485 \phi^{5} \\
g_{\sigma}^{\text {expt }} & =\frac{1}{4 \phi}\left(\frac{1+\phi+\phi^{2}-2 / 3 \phi^{3}-2 / 3 \phi^{4}}{(1-\phi)^{4}}-1\right) \\
\alpha & =\frac{1}{2 a}\left(44.554+79.868 \phi+116.432 \phi^{2}-44.652 \cdot e^{(2 \phi)}\right) \\
\beta & =\frac{1}{2 a}\left(-5.022+5.857 \phi+5.089 \cdot e^{(-4 \phi)}\right) \\
\omega & =\frac{1}{2 a}\left(-0.682 e^{(-24.697 \phi)}+4.720+4.450 \phi\right) \\
\kappa & =\frac{1}{2 a}\left(4.674 \cdot e^{(-3.935 \phi)}+3.536 \cdot e^{(-56.270 \phi)}\right) \\
\mu & =\frac{\phi}{2 a(1-\phi)}\left(-1+\frac{d}{2 \phi}+\frac{\phi}{d}\right) \\
d & =\left\{2 \phi\left[\phi^{2}-3 \phi-3+\sqrt{3\left(\phi^{4}-2 \phi^{3}+\phi^{2}+6 \phi+3\right)}\right]\right\}^{1 / 3} .
\end{aligned}
$$

Finally, on the basis of Eq. (62), the unknown coefficient $\gamma$ can be found by solving the following equation:

$$
f_{1}+f_{2}=0
$$

where

$$
\begin{aligned}
& f_{1}=\frac{A \cdot e^{\mu\left(r^{*}-2 a\right)}}{\left(r^{*}\right)^{2}} \cdot\left(\mu \cdot r^{*}-1\right) \\
& f_{2}=\frac{B}{\left(r^{*}\right)^{2}}\left\{\cos \left(\beta\left[r^{*}-2 a\right]+\gamma\right) \cdot\left(\alpha \cdot r^{*}-1\right)-\beta \cdot r^{*} \sin \left(\beta\left[r^{*}-2 a\right]+\gamma\right)\right\} .
\end{aligned}
$$

\section{References}

1. Eshelby, J.D.: The determination of the elastic field of an ellipsoidal inclusion, and related problems. Proc. R. Soc. Lond. A241, 376-396 (1957)

2. Qu, J., Cherkaoui, M.: Fundamentals of Micromechanics of Solids. Wiley, London (2006)

3. Moschovidis, Z.A., Mura, T.: Two-ellipsoidal inhomogeneities by the equivalent inclusion method. ASME J. Appl. Mech. 42, 847-852 (1975)

4. Ju, J.W., Chen, T.M.: Micromechanics and effective moduli of elastic composites containing randomly dispersed ellipsoidal inhomogeneities. Acta Mech. 103, 103-121 (1994)

5. Ju, J.W., Chen, T.M.: Effective elastic moduli of two-phase composites containing randomly dispersed spherical inhomogeneities. Acta Mech. 103, 123-144 (1994)

6. Ju, J.W., Chen, T.M.: Micromechanics and effective elastoplastic behavior of two-phase metal matrix composites. ASME J. Eng. Mech. 116, 310-318 (1994)

7. Ju, J.W., Zhang, X.D.: Micromechanics and effective transverse elastic moduli of composites with randomly located aligned circular fibers. Int. J. Solids Struct. 35, 941-960 (1998) 
8. Ju, J.W., Zhang, X.D.: Effective elastoplastic behavior of ductile matrix composites containing randomly located aligned circular fibers. Int. J. Solids Struct. 38, 4045-4069 (2001)

9. Lin, P.J., Ju, J.W.: Effective elastic moduli of three-phase composites with randomly located and interacting spherical particles of distinct properties. Acta Mech. 208, 11-26 (2009)

10. Mura, T.: Micromechanics of Defects in Solids. 2nd edn. Martinus Nijhoff Publishers, Dordrecht (1987)

11. Nemat-Nasser, S., Hori, M.: Micromechanics: overall properties heterogeneous materials. Elsevier, The Netherlands (1993)

12. Ju, J.W., Sun, L.Z.: A novel formulation for the exterior-point Eshelby's tensor of an ellipsoidal inclusion. ASME J. Appl. Mech. 66, 570-574 (1999)

13. Ju, J.W., Sun, L.Z.: Effective elastoplastic behavior of metal matrix composites containing randomly located aligned spheroidal inhomogeneities. Part I: micromechanics-based formulation. Int. J. Solids Struct. 38, 183-201 (2001)

14. Sun, L.Z., Ju, J.W.: Effective elastoplastic behavior of metal matrix composites containing randomly located aligned spheroidal inhomogeneities. Part II: applications. Int. J. Solids Struct. 38, 203-225 (2001)

15. Sun, L.Z., Ju, J.W.: Elastoplastic modeling of metal matrix composites containing randomly located and oriented spheroidal particles. ASME J. Appl. Mech. 71, 774-785 (2004)

16. Ju, J.W., Yanase, K.: Micromechanical elastoplastic damage mechanics for elliptical fiber reinforced composites with progressive partial fiber debonding. Int. J. Damage. Mech. 18, 639-668 (2009)

17. Hu, G.K., Weng, G.J.: Influence of thermal residual stress on the composite macroscopic behavior. Mech. Mater. 27, 229240 (1998)

18. Liu, H.T., Sun, L.Z.: Effects of thermal residual stress on effective elastoplastic behavior of metal matrix composites. Int. J. Solids Struct. 41, 2189-2203 (2004)

19. Ju, J.W., Yanase, K.: Elastoplastic damage micromechanics for elliptical fiber composites with progressive partial fiber debonding and thermal residual stresses. Theor. Appl. Mech. 35, 137-170 (2008)

20. Tanaka, K., Mori, T.: Note on volume integral of the elastic field around an ellipsoid inclusion. J. Elasticity. 2, 199-200 (1972)

21. Li, S., Wang, G.: Introduction to micromechanics and nanomechanics. World Scientific Publishing Co. Pte. Ltd, Singapore (2008)

22. Trokhymchuk, A., Nezbeda, I., Jirsák, J., Henderson, D.: Hard-sphere radial distribution function again. J. Chem. Phys. 123, 024501 (2005)

23. Mori, T., Tanaka, K.: Average stress in matrix and average elastic energy of materials with misfitting inclusions. Acta Metall. 21, 571-574 (1973)

24. Zhao, Y.H., Tandom, G.P., Weng, G.J.: Elastic moduli for a class of porous materials. Acta Mech. 76, 105-131 (1989)

25. Hashin, Z., Shtrikman, S.: A variational approach to the theory of the elastic behavior of multiphase materials. J. Mech. Phys. Solids 11, 127-140 (1963)

26. Weng, G.J.: The theoretical connection between Mori-Tanaka's theory and the Hashin-Shtrikman-Walpole bounds. Int. J. Eng. Sci. 28, 1111-1120 (1990)

27. Smith, J.C.: Experimental values for the elastic constants of a particulate-filled glassy polymer. J. Res. NBS. 80, 45-49 (1976)

28. Richard, T.G.: The mechanical behavior of a solid microsphere filled composite. J. Comp. Mat. 9, 108-113 (1975)

29. Paul., B.: Trans. AIME 218, 36-41 (1960)

30. Walsh, J.B., Brace, W.F., England, W.F.: Effect of porosity on compressibility of glass. J. Am. Ceram. Soc. 48, 605-608 (1965) 